\title{
Basal Ganglia: Mechanisms for Action Selection
}

Link to publication record in Manchester Research Explorer

\section{Citation for published version (APA):}

Humphries, M. D., Jaeger, D. (Ed.), \& Jung, R. (Ed.) (2014). Basal Ganglia: Mechanisms for Action Selection. In Encyclopedia of Computational Neuroscience (pp. 1-7). Springer Nature.

\section{Published in:}

Encyclopedia of Computational Neuroscience

\section{Citing this paper}

Please note that where the full-text provided on Manchester Research Explorer is the Author Accepted Manuscript or Proof version this may differ from the final Published version. If citing, it is advised that you check and use the publisher's definitive version.

\section{General rights}

Copyright and moral rights for the publications made accessible in the Research Explorer are retained by the authors and/or other copyright owners and it is a condition of accessing publications that users recognise and abide by the legal requirements associated with these rights.

\section{Takedown policy}

If you believe that this document breaches copyright please refer to the University of Manchester's Takedown Procedures [http://man.ac.uk/04Y6Bo] or contact uml.scholarlycommunications@manchester.ac.uk providing relevant details, so we can investigate your claim.

\section{OPEN ACCESS}




\section{Basal Ganglia: Mechanisms for Action Selection}

Mark D Humphries*

Faculty of Life Sciences, University of Manchester, Manchester, UK

\section{Definition}

The basal ganglia are an interconnected circuit of fore- and midbrain nuclei common to all extant vertebrates, including the lamprey, which diverged from the main vertebrate line approximately 560 MYA. Moreover, there are analogous structures in birds and bony fish. The preservation of this circuit implies a crucial operation that solves a problem common to all vertebrates. While many functions for the basal ganglia have been proposed, our current best theory to unify these ideas is that this circuit solves the common problem of action selection. Informally put, this is the problem of how to decide what to do next.

\section{Detailed Description}

\section{Why Action Selection?}

The necessity of a mechanism for action selection is imposed by the strong constraint that complex animals have a final common motor pathway: the connections of the spinal cord and the number of muscle groups limit the set of actions that can be expressed simultaneously. Some mechanism is required to reduce the repertoire of currently available actions to just those actions capable of simultaneous expression. Given the constraints of biological tissue, a central rather than distributed selection mechanism is likely the preferred solution implemented by a neural substrate (Prescott et al. 1999). Briefly, the argument runs as follows. Typically, distributed selection mechanisms, formed by reciprocal, inhibitory links between $n$ behavior-representing nodes, contain $n(n-1)$ links and grow as $2 n$ for every additional node. By contrast, a central selection device which is reciprocally connected with all $n$ nodes (thus allowing control over the expression of each node's represented behavior) requires just $2 n$ links and grows by 2 for each additional node. Thus, a central selection device is more economical in both the number of connections required and the cost of adding nodes. Such economy of wiring appears to be a priority for the central nervous system (Cherniak 1994).

The basal ganglia appear to fulfill the criteria for such a central mechanism (Redgrave et al. 1999). The main input nucleus, the striatum, receives input from every region of cortex, from primary visual, auditory, and somatosensory cortex, through motor cortices, to the subregions of prefrontal cortex. It is thus in a position to integrate sensory information, current motor commands, planning, and goals into representations of the relative importance, or salience, of each action. The output of the basal ganglia targets regions in the thalamus and brain stem that underpin the execution of different actions. The basal ganglia are thus also in a position to directly control the activity in neural systems responsible for executing actions.

The idea that the basal ganglia perform action selection is also a potential solution to the longstanding paradox of their role in controlling movement (Marsden and Obeso 1994). Activity in the

\footnotetext{
*Email: mark.humphries@manchester.ac.uk
} 


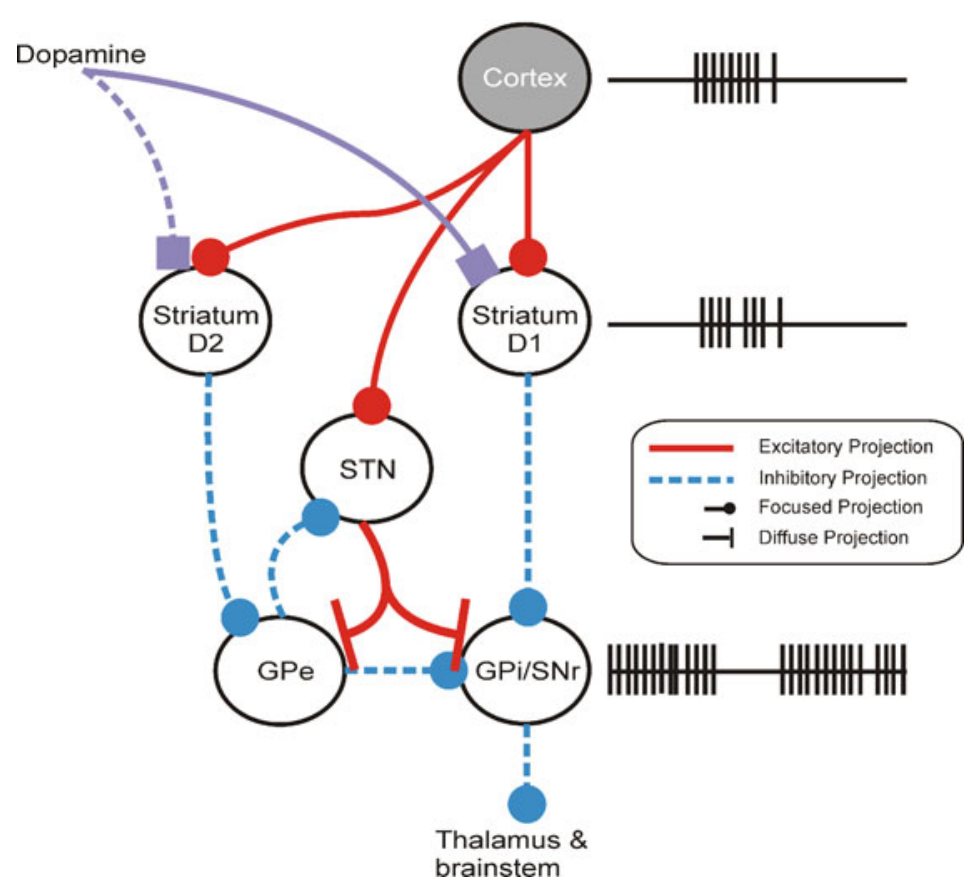

Fig. 1 Basal ganglia circuit and selection. The striatum receives input from most of cortex and many thalamic nuclei; a subset of the same regions project to the subthalamic nucleus (STN). The striatum is split into two populations of projection neurons according to whether they predominantly express the D1 or D2 receptor for dopamine: the populations and their targets respectively form the "direct" and "indirect" pathways. The globus pallidus external segment (GPe) projects within the basal ganglia; the globus pallidus internal segment (GPi) and substantia nigra pars reticulata ( $\mathrm{SNr}$ ) project to targets in the brain stem and thalamus. The cartoon spike-trains illustrate the basic process of disinhibition in the "direct" pathway: continuous firing from the GPi/SNr tonically inhibits their targets; brief phasic activity in striatum, driven by coordinated cortical activity, can pause that tonic inhibition, allowing the targets to fire

basal ganglia is strongly correlated with movement onset and parameters of that movement, and stimulation of regions of the striatum can evoke discrete, smooth limb movements (Alexander and DeLong 1985). Yet lesions of basal ganglia nuclei in animal studies or in Parkinson's disease patients to alleviate their symptoms do not clearly impair the initiation of movement (Marsden and Obeso 1994). The theory that the basal ganglia are necessary to control the choice of actions explains how movement-related activity can arise yet not be needed to directly generate movement.

\section{How Can the Basal Ganglia Circuit "Select"?}

While the basal ganglia's inputs and outputs position these nuclei to potentially act as a central selection mechanism, the key evidence in favor of this idea is that their internal circuitry is seemingly designed to implement a selection process. Figure 1 illustrates the basic circuitry of the basal ganglia as currently understood. The original proposals for how the internal circuitry of the basal ganglia may implement a selection process envisioned a division of labor between the "direct" and "indirect" pathways (Albin et al. 1989; DeLong 1990; Alexander and Crutcher 1990). The direct pathway was proposed to be responsible for selection through a process of disinhibition (Chevalier and Deniau 1990), illustrated in Fig. 1. The output nuclei of the basal ganglia are continuously active, providing a source of constant (or "tonic") inhibition to their targets. A sufficiently strong input signal from cortex can elicit activity in a population of projection neurons in the striatum, which in turn converge on and temporarily inhibit the firing of neurons in the output nuclei. This pause in tonic firing is then interpreted as the signal for selection. 
This disinhibition process has been worked out in detail for the control of voluntary eye movements, or saccades, in primates (see Hikosaka et al. 2000). Phasic activity in the caudate region of the striatum precedes saccade initiation and is time-locked to a decrease in activity in a subset of $\mathrm{SNr}$ neurons. The $\mathrm{SNr}$ projects to the intermediate layers of the superior colliculus, which contains a retinotopic map - neurons are laid out in a sheet that represents coordinates of gaze direction. A buildup of neural activity on this map drives the brain stem circuitry that controls the eye muscles and moves gaze to the location signaled by the map. The pause in $\mathrm{SNr}$ activity precedes and appears to be required for the buildup of activity in the retinotopic map of the superior colliculus. Conversely, continuously inhibiting $\mathrm{SNr}$ activity results in the inability to suppress saccades, as though every eye movement motor command was being executed (Hikosaka and Wurtz 1985). Thus, the direct striatum-SNr pathway likely controls the selection of saccade direction through a process of disinhibition.

\section{Multiple Mechanisms Contributing to Action Selection}

Computational models have provided quantitative tests of these ideas, revealed the further necessary mechanisms for successful selection, and shown how these mechanisms are implemented by components of the basal ganglia circuit. The box-and-arrow model accounts of "direct" pathway control of selection focussed on the flow of signals necessary to select one input and so omitted two crucial aspects of a selection process: how to resolve the competition between multiple actionrepresenting inputs and how to switch from a currently selected action to another, more salient, action.

\section{Competition}

To be able to resolve a competition between actions first requires a substrate for separately representing each of those actions. A long-standing concept in basal ganglia research is the existence of parallel loops running through the basal ganglia (Alexander et al. 1986; Middleton and Strick 2000). At the macroscopic level, independent domains of the basal ganglia have been identified by the closed send-and-return loops originating in broad cortical regions (Alexander et al. 1986). Each macroscopic domain is thought to subdivide into a further large set of discrete parallel loops, termed channels here to distinguish them from the macroscopic-scale loops. For example, the somatotopic map found within the striatal motor domain is maintained throughout the basal ganglia's intrinsic circuitry, such that there are separate channels for arm, leg, and face representations (Alexander and Crutcher 1990; Romanelli et al. 2005). Similar topographic maps have been proposed for the other macroscopic loops (Alexander and Crutcher 1990). Moreover, within these limb representations, there may be additional discrete channels corresponding to particular movements, demonstrated in striatum by microstimulation (Alexander and DeLong 1985) and markers for metabolic activity during behavior (Brown and Sharp 1995), and to repeated representations of body map locations (Brown et al. 1998). Computational models of the basal ganglia have proposed that it is these channels which form the basis for action representation, and so the resolution of competition is expressed by the selection of a particular channel in the output of the basal ganglia (Gurney et al. 2001a).

The basal ganglia circuit contains a number of mechanisms potentially suited to resolving competition between competing channels. One candidate mechanism is the inhibitory network formed by the local axon collaterals of the projection neurons in striatum, which could underpin a winner-takes-all competition between input signals (Alexander and Wickens 1993). A problem with this idea is that these feedback connections are weak and asymmetric so that a classical winner-takes-all competition is unlikely to be supported (Plenz 2003). Even if it is, because of the 


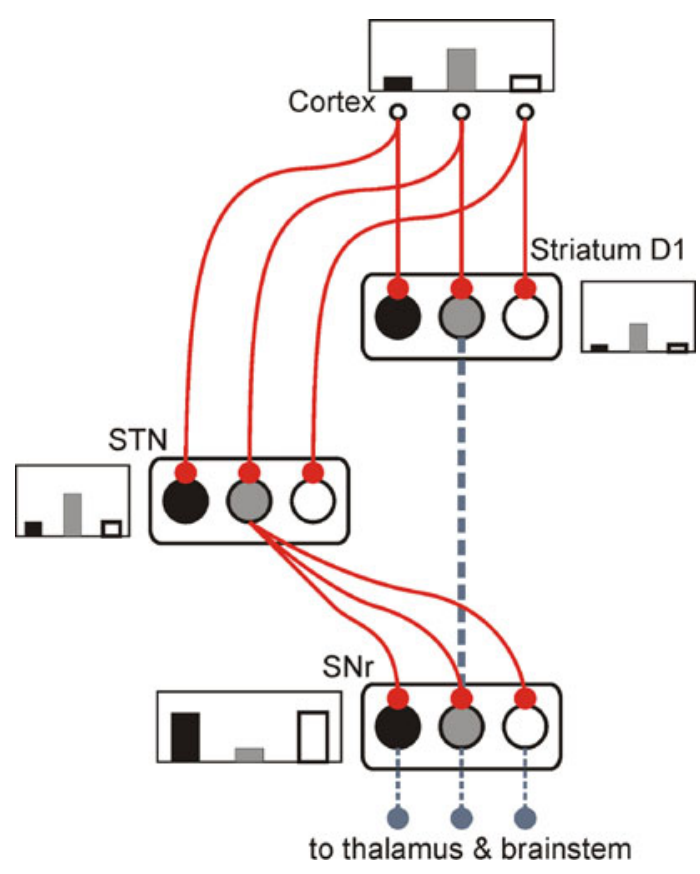

Fig. 2 Competition resolution and switching by the basal ganglia. Actions are thought to be represented in parallel loops or channels running throughout the basal ganglia, with each action's relative salience computed by inputs from cortex. The bars in each box are cartoons of activity of each population in each structure. Each striatal population projects to a corresponding SNr/GPi population, providing focussed inhibitory input; STN output is comparatively diffuse, projecting across neighboring channels and providing diffuse excitatory input. The result is an off-center, onsurround network in $\mathrm{SNr} / \mathrm{GPi}$, where the strongest signal from among the striatal populations is able to inhibit the firing of its target $\mathrm{SNr} / \mathrm{GPi}$ population, while at the same time the combined excitatory output of the STN counteracts the effect of the other inhibitory signals from striatum

range of the local axon collaterals, this interaction can only extend over a short distance in the striatum, on the order of 200-300 $\mu \mathrm{m}$ (Wickens 1997; Humphries et al. 2010). Consequently, any dynamics within striatum are likely only to resolve very local competitions between cortical inputs (Gurney et al. 2001b).

The competition between a set of channels most likely takes place at the inputs to the basal ganglia output nuclei, the SNr and GPi. As shown in Fig. 2, each population of striatal neurons projects to a corresponding population of $\mathrm{SNr}$ or GPi neurons, but the output of each population of STN neurons also contacts neighboring channels in SNr and GPi. This creates an off-center, onsurround network ideal for resolving competition between action signals (Mink 1996; Gurney et al. 2001a; Humphries et al. 2006; Leblois et al. 2006) as illustrated in Fig. 2.

A key insight from computational models is that the on-surround effect of the combined STN output can lead to SNr/GPi activity in many channels increasing above their tonic rate at the time of action selection - a phenomenon termed contrast enhancement. This makes functional sense: an enhanced tonic inhibitory signal being output from most channels indicates a definite nonselection of that action. However, when observed in experimental data, such increases in activity, timelocked to movement, have been interpreted as encoding that movement. By contrast, the models show how an increase in $\mathrm{SNr} / \mathrm{GPi}$ neuron output time-locked to an action does not encode that action, but rather encodes the simultaneous nonselection of another potential action. Moreover, the models show that the number of neurons with such increased activity, time-locked to an action, will be in the majority and hence will appear more frequently during neural recordings (Humphries et al. 2006). 


\title{
Switching
}

Once action selection has been achieved, then action switching is required: the sudden availability of a more salient action through changes in the environment or the animal's needs should terminate the ongoing action and then switch to selecting the new, more salient alternative. Computational models have again shown how the same off-center, on-surround design naturally handles this: the new input further increases the STN output, canceling selection of the current action, while the corresponding new, strong inhibitory signal from striatum inhibits its target population in GPi/SNR (Gurney et al. 2001b; Humphries and Gurney 2002). Embedding these models in mobile robots has shown how smooth behavioral sequences naturally emerge from the changing saliences of each action, without the need for explicit storage of sequences (Khamassi et al. 2005; Prescott et al. 2006).

\section{The "Indirect" Pathway: Prevention of Selection and Scaling of Selection}

The contribution of the "indirect" pathway to selection has been more difficult to unravel. The original box-and-arrow models proposed that this pathway acts to counteract the selection of an action: increased inhibition of the GPe by its striatal inputs would lead to enhanced STN output to $\mathrm{SNr} / \mathrm{GPi}$, thereby counteracting inhibition they were receiving in the direct pathway (Alexander and Crutcher 1990). Consequently, it was proposed that any imbalance between the relative strengths of the direct and indirect pathways could lead to movement disorders (Albin et al. 1989). Recent experimental studies have shown how selectively activating the striatal D1 or D2 projection neuron populations does indeed lead to opposite effects on behavior: stimulation of D1 projection neurons facilitates behavior, whereas stimulation of D2 neurons suppresses ongoing behavior (Kravitz et al. 2010).

The computational models of Frank and colleagues (Frank et al. 2004; Frank 2005) have extended these ideas by proposing that recruitment of these "go" and "no-go" pathways by cortical input during learning would depend on the level of available dopamine. They showed these models could account for a range of learning biases exhibited by Parkinson's disease patients, providing support for the go versus no-go competition between the direct and indirect pathways.

A further mystery was the role of STN feedback to the GPe (Fig. 1). An analysis by Gurney et al. (2001b) showed that this negative feedback loop provided capacity scaling for action selection. Without a loop between STN and GPe, the diffuse STN output means that the total excitatory input to the SNr/GPi would grow in proportion to the number of active inputs to the basal ganglia; with a sufficient number of inputs, the excitatory input would grow large enough to overwrite all focussed inhibition from the striatum and prevent any selection. However, the negative feedback loop ensures that the STN output is scaled to the number of active inputs: the more the inputs, the more the STN output tries to grow, but is held in check by the GPe output which is itself a function of the STN output. In this way, the basal ganglia's capacity can scale and so can operate as a selection mechanism independently of the number of currently competing channels.

\section{Cross-References}

\author{
- Basal Ganglia: Overview \\ Decision Making: Overview \\ - Reinforcement-Learning in Cortical Networks
}




\section{References}

Albin RL, Young AB, Penney JB (1989) The functional anatomy of basal ganglia disorders. Trends Neurosci 12:366-375

Alexander GE, Crutcher MD (1990) Functional architecture of basal ganglia circuits: neural substrates of parallel processing. Trends Neurosci 13:266-272

Alexander GE, DeLong MR (1985) Microstimulation of the primate neostriatum. I. Physiological properties of striatal microexcitable zones. J Neurophysiol 53:1401-1416

Alexander ME, Wickens JR (1993) Analysis of striatal dynamics: the existence of two modes of behaviour. J Theor Biol 163:413-438

Alexander GE, DeLong MR, Strick PL (1986) Parallel organization of functionally segregated circuits linking basal ganglia and cortex. Annu Rev Neurosci 9:357-381

Brown LL, Sharp FR (1995) Metabolic mapping of rat striatum: somatotopic organization of sensorimotor activity. Brain Res 686:207-222

Brown LL, Smith DM, Goldbloom LM (1998) Organizing principles of cortical integration in the rat neostriatum: corticostriate map of the body surface is an ordered lattice of curved laminae and radial points. J Comp Neurol 392:468-488

Cherniak C (1994) Component placement optimization in the brain. J Neurosci 14:2418-2427

Chevalier G, Deniau JM (1990) Disinhibition as a basic process in the expression of striatal function. Trends Neurosci 13:277-280

DeLong MR (1990) Primate models of movement disorders of basal ganglia origin. Trends Neurosci 13:281-28

Frank MJ (2005) Dynamic dopamine modulation in the basal ganglia: a neurocomputational account of cognitive deficits in medicated and nonmedicated Parkinsonism. J Cogn Neurosci 17:51-72

Frank MJ, Seeberger LC, O’Reilly RC (2004) By carrot or by stick: cognitive reinforcement learning in parkinsonism. Science 306:1940-1943

Gurney K, Prescott TJ, Redgrave P (2001a) A computational model of action selection in the basal ganglia I: a new functional anatomy. Biol Cyber 85:401-410

Gurney K, Prescott TJ, Redgrave P (2001b) A computational model of action selection in the basal ganglia II: analysis and simulation of behaviour. Biol Cyber 85:411-423

Hikosaka O, Wurtz RH (1985) Modification of saccadic eye movements by GABA-related substances. II. Effects of muscimol in monkey substantia nigra pars reticulata. J Neurophysiol 53:292-308

Hikosaka O, Takikawa Y, Kawagoe R (2000) Role of the basal ganglia in the control of purposive saccadic eye movements. Physiol Rev 80:953-978

Humphries MD, Gurney KN (2002) The role of intra-thalamic and thalamocortical circuits in action selection. Netw Comput Neural Syst 13:131-156

Humphries MD, Stewart RD, Gurney KN (2006) A physiologically plausible model of action selection and oscillatory activity in the basal ganglia. J Neurosci 26:12921-12942

Humphries MD, Wood R, Gurney K (2010) Reconstructing the three-dimensional GABAergic microcircuit of the striatum. PLoS Comput Biol 6:e1001011

Khamassi M, Lacheze L, Girard B, Berthoz A, Guillot A (2005) Actor-critic models of reinforcement learning in the basal ganglia: from natural to artificial rats. Adapt Behav 13:131-148

Kravitz AV, Freeze BS, Parker PRL, Kay K, Thwin MT, Deisseroth K, Kreitzer AC (2010) Regulation of parkinsonian motor behaviours by optogenetic control of basal ganglia circuitry. Nature 466:622-626 
Leblois A, Boraud T, Meissner W, Bergman H, Hansel D (2006) Competition between feedback loops underlies normal and pathological dynamics in the basal ganglia. $J$ Neurosci 26:3567-3583

Marsden CD, Obeso JA (1994) The functions of the basal ganglia and the paradox of stereotaxic surgery in Parkinson's disease. Brain 117:877-897

Middleton FA, Strick PL (2000) Basal ganglia and cerebellar loops: motor and cognitive circuits. Brain Res Brain Res Rev 31:236-250

Mink JW (1996) The basal ganglia: focused selection and inhibition of competing motor programs. Prog Neurobiol 50:381-425

Plenz D (2003) When inhibition goes incognito: feedback interaction between spiny projection neurons in striatal function. Trends Neurosci 26:436-443

Prescott TJ, Redgrave P, Gurney K (1999) Layered control architectures in robots and vertebrates. Adapt Behav 7:99-127

Prescott TJ, Montes-Gonzalez FM, Gurney K, Humphries MD, Redgrave P (2006) A robot model of the basal ganglia: behavior and intrinsic processing. Neural Netw 19:31-61

Redgrave P, Prescott TJ, Gurney K (1999) The basal ganglia: a vertebrate solution to the selection problem? Neuroscience 89:1009-1023

Romanelli P, Esposito V, Schaal DW, Heit G (2005) Somatotopy in the basal ganglia: experimental and clinical evidence for segregated sensorimotor channels. Brain Res Brain Res Rev 48:112-128

Wickens J (1997) Basal ganglia: structure and computations. Netw Comput Neural Syst 8: R77-R109 